\title{
On the geometry of Poincaré's problem for one-dimensional projective foliations
}

\author{
MARCIO G. SOARES* \\ Departamento de Matemática, ICEx, UFMG - 31270-901 Belo Horizonte, Brazil \\ Manuscript received on June 28, 2001; accepted for publication on July 18, 2001.
}

\begin{abstract}
We consider the question of relating extrinsic geometric characters of a smooth irreducible complex projective variety, which is invariant by a one-dimensional holomorphic foliation on a complex projective space, to geometric objects associated to the foliation.
\end{abstract}

Key words: holomorphic foliations, invariant varieties, polar classes, degrees.

\section{INTRODUCTION}

H. Poincare treated, in (1891), the question of bounding the degree of an algebraic curve, which is a solution of a foliation $\mathcal{F}$ on $\mathbb{P}_{\mathbb{C}}^{2}$ with rational first integral, in terms of the degree of the foliation. This problem has been considered more recently in the following formulation: to bound the degree of an irreducible algebraic curve $S$, invariant by a foliation $\mathcal{F}$ on $\mathbb{P}_{\mathbb{C}}^{2}$, in terms of the degree of the foliation.

Simple examples show that, when $S$ is a dicritical separatrix of $\mathcal{F}$, the search for a positive solution to the problem is meaningless. The obstruction in this case was given by M. Brunella in (1997), and reads: the number $\int_{S} c_{1}\left(N_{\mathcal{F}}\right)-S \cdot S$ may be negative if $S$ is a dicritical separatrix (here, $N_{\mathcal{F}}$ is the normal bundle of the foliation). More than that, A. Lins Neto constructs, in (2000), some remarkable families of foliations on $\mathbb{P}_{\mathbb{C}}^{2}$ providing counterexamples for this problem, all involving singular separatrices and dicritical singularities.

However, as was shown in (Brunella 1997), when $S$ is a non-dicritical separatrix, the number $\int_{S} c_{1}\left(N_{\mathcal{F}}\right)-S \cdot S$ is nonnegative and, in $\mathbb{P}_{\mathbb{C}}^{2}$, this means $d^{0}(\mathcal{F})+2 \geq d^{0}(S)$, where $d^{0}(\mathcal{F})$ and $d^{0}(S)$ are the degrees of the foliation and of the curve, respectively. Another solution to the problem, in the non-dicritical case, was given by M.M. Carnicer in (1994), using resolution of singularities.

\footnotetext{
* Member of Academia Brasileira de Ciências

E-mail: msoares@math.ufmg.br
} 
Let us now consider one-dimensional holomorphic foliations on $\mathbb{P}_{\mathbb{C}}^{n}, n \geq 2$, that is, morphisms $\mathcal{F}: \mathcal{O}(m) \longrightarrow \mathrm{TP}_{\mathbb{C}}^{n}, m \in \mathbb{Z}, m \leq 1$, with singular set of codimension at least 2 . We write $m=1-d^{0}(\mathcal{F})$ and call $d^{0}(\mathcal{F}) \geq 0$ the degree of $\mathcal{F}$. From now on we will consider $d^{0}(\mathcal{F}) \geq 2$. This is the characteristic number associated to the foliation.

On the other hand, if we consider $\mathcal{F}$-invariant algebraic varieties $\mathbf{V} \stackrel{\mathbf{i}}{\longrightarrow} \mathbb{P}_{\mathbb{C}}^{n}$, it is natural to consider other characters associated to $\mathbf{V}$, not just its degree. This is the point of view we address. More precisely, we pose the question of relating extrinsic geometric characters of $\mathbf{V}$ to geometric objects associated to $\mathcal{F}$.

This approach produces some interesting results. Let us illustrate the two-dimensional situation. Suppose we have an $\mathcal{F}$-invariant irreducible plane curve $S$. We associate to $\mathcal{F}$ a tangency divisor $\mathcal{D}_{\mathcal{H}}$ (depending on a pencil $\mathcal{H}$ ), which is a curve of degree $d^{0}(\mathcal{F})+1$ and contains the first polar locus of $S$. Computing degrees we arrive at $d^{0}(S) \leq d^{0}(\mathcal{F})+2$ in case $S$ is smooth, and at $d^{0}(S)\left(d^{0}(S)-1\right)-\sum_{p \in \operatorname{sing}(S)}\left(\mu_{p}-1\right) \leq\left(d^{0}(\mathcal{F})+1\right) d^{0}(S)$ in case $S$ is singular, where $\mu_{p}$ is the Milnor number of $S$ at $p$. This allows us to recover a result of D. Cerveau and A. Lins Neto (1991), which states that if $S$ has only nodes as singularities, then $d^{0}(S) \leq d^{0}(\mathcal{F})+2$, regardless of the singularities of $\mathcal{F}$ being dicritical or non-dicritical.

In the higher dimensional situation, we obtain relations among polar classes of $\mathcal{F}$-invariant smooth varieties and the degree of the foliation.

\section{THE TANGENCY DIVISOR OF $\mathcal{F}$ WITH RESPECT TO A PENCIL}

Let $\mathcal{F}$ be a one-dimensional holomorphic foliation on $\mathbb{P}_{\mathbb{C}}^{n}$ of degree $d^{0}(\mathcal{F}) \geq 2$, with singular set of codimension at least 2 . We associate a tangency divisor to $\mathcal{F}$ as follows:

Choose affine coordinates $\left(z_{1}, \ldots, z_{n}\right)$ such that the hyperplane at infinity, with respect to these, is not $\mathcal{F}$-invariant, and let $X=g R+\sum_{i=1}^{n} Y_{i} \frac{\partial}{\partial z_{i}}$ be a vector field representing $\mathcal{F}$, where $R=\sum_{i=1}^{n} z_{i} \frac{\partial}{\partial z_{i}}, g\left(z_{1}, \ldots, z_{n}\right) \not \equiv 0$ is homogeneous of degree $d^{0}(\mathcal{F})$ and $Y_{i}\left(z_{1}, \ldots, z_{n}\right)$ is a polynomial of degree $\leq d^{0}(\mathcal{F}), 1 \leq i \leq n$. Let $H$ be a generic hyperplane in $\mathbb{P}_{\mathbb{C}}^{n}$. Then, the set of points in $H$ which are either singular points of $\mathcal{F}$ or at which the leaves of $\mathcal{F}$ are not transversal to $H$ is an algebraic set, noted $\operatorname{tang}\left(H, \mathcal{F}\right.$ ), of dimension $n-2$ and degree $d^{0}(\mathcal{F})$ (observe that $g\left(z_{1}, \ldots, z_{n}\right)=0$ is precisely $\left.\tan g\left(H_{\infty}, \mathcal{F}\right)\right)$.

Definition. Consider a pencil of hyperplanes $\mathcal{H}=\left\{H_{t}\right\}_{t \in \mathbb{P}_{\mathbb{C}}^{1}}$, with axis $L^{n-2}$. The tangency divisor of $\mathcal{F}$ with respect to $\mathcal{H}$ is

$$
\mathcal{D}_{\mathcal{H}}=\bigcup_{t \in \mathbb{P}_{\mathbb{C}}^{1}} \operatorname{tang}\left(H_{t}, \mathcal{F}\right)
$$

LeMma 2.1. $\mathcal{D}_{\mathcal{H}}$ is a (possibly singular) hypersurface of degree $d^{0}(\mathcal{F})+1$.

Proof. Let $p$ be a point in $L^{n-2}$, the axis of the pencil. If $p \in \sin g(\mathcal{F})$ then $p$ is necessarily in $\mathcal{D}_{\mathcal{H}}$, otherwise $p$ is a regular point of $\mathcal{F}$. In this case, if $\mathcal{L}$ is the leaf of $\mathcal{F}$ through $p$, then either $\mathrm{T}_{p} \mathcal{L} \subset L^{n-2}$ or, $\mathrm{T}_{p} \mathcal{L}$ together with $L^{n-2}$ determine a hyperplane $H_{\alpha} \in \mathcal{H}$, and hence we have 
$p \in \operatorname{tang}\left(H_{\alpha}, \mathcal{F}\right) \subset \mathcal{D}_{\mathcal{H}}$, so that $L^{n-2} \subset \mathcal{D}_{\mathcal{H}}$. Now, let $p \in L^{n-2}$ be a regular point of $\mathcal{F}$ and choose a generic line $\ell$, transverse to $L^{n-2}$, passing through $p$ and such that $L^{n-2}$ and $\ell$ determine a hyperplane $H_{\beta}$, distinct from $H_{\alpha}$. This line $\ell$ meets $\mathcal{D}_{\mathcal{H}}$ at $p$ and at $d^{0}(\mathcal{F})$ further points, counting multiplicities, corresponding to the intersections of $\ell$ with $\operatorname{tang}\left(H_{\beta}, \mathcal{F}\right)$. Hence $\mathcal{D}_{\mathcal{H}}$ has degree $d^{0}(\mathcal{F})+1$.

EXAMPLE. If we consider the two-dimensional Jouanolou's example

$$
\begin{gathered}
\dot{x}=y^{d^{0}(\mathcal{F})}-x^{d^{0}(\mathcal{F})+1} \\
\dot{y}=1-y x^{d^{0}(\mathcal{F})}
\end{gathered}
$$

and the pencil $\mathcal{H}=\left\{(a t, b t): t \in \mathbb{C},(a: b) \in \mathbb{P}_{\mathbb{C}}^{1}\right\}$, a straightforward manipulation shows that $\mathcal{D}_{\mathcal{H}}$ is given, in homogeneous coordinates $(X: Y: Z)$ in $\mathbb{P}_{\mathbb{C}}^{2}$, by

$$
Y^{d^{0}(\mathcal{F})+1}-X Z^{d^{0}(\mathcal{F})}=0 .
$$

\section{$3 \quad \mathcal{F}$-INVARIANT SMOOTH IRREDUCIBLE VARIETIES}

Let us recall some facts about polar varieties and classes (Fulton 1984). If $\mathbf{V} \stackrel{\mathbf{i}}{\longrightarrow} \mathbb{P}_{\mathbb{C}}^{n}$ is a smooth irreducible algebraic subvariety of $\mathbb{P}_{\mathbb{C}}^{n}$, of dimension $n-k$, and $L^{k+j-2}$ is a linear subspace, then the $\mathrm{j}$-th polar locus of $\mathbf{V}$ is defined by

$$
\mathcal{P}_{j}(\mathbf{V})=\left\{q \in \mathbf{V} \mid \operatorname{dim}\left(\mathrm{T}_{q} \mathbf{V} \cap L^{k+j-2}\right) \geq j-1\right\}
$$

for $0 \leq j \leq n-k$. If $L^{k+j-2}$ is a generic subspace, the codimension of $\mathcal{P}_{j}(\mathbf{V})$ in $\mathbf{V}$ is precisely $j$. The j-th class, $\varrho_{j}(\mathbf{V})$, of $\mathbf{V}$ is the degree of $\mathcal{P}_{j}(\mathbf{V})$ and, since the cycle associated to $\mathcal{P}_{j}(\mathbf{V})$ is

$$
\left[\mathcal{P}_{j}(\mathbf{V})\right]=\sum_{i=0}^{j}(-1)^{i}\left(\begin{array}{c}
n-k-i+1 \\
j-i
\end{array}\right) c_{i}(\mathbf{V}) c_{1}\left(\mathbf{i}^{*} \mathcal{O}(1)\right)^{j-i}
$$

we have

$$
\varrho_{j}(\mathbf{V})=\int_{\mathbf{V}} \sum_{i=0}^{j}(-1)^{i}\left(\begin{array}{c}
n-k-i+1 \\
j-i
\end{array}\right) c_{i}(\mathbf{V}) c_{1}\left(\mathbf{i}^{*} \mathcal{O}(1)\right)^{n-k-i} \quad, \quad 0 \leq j \leq n-k .
$$

Lemma 3.1. Let $\mathbf{V}$ be a smooth irreducible algebraic variety of dimension $n-k, \mathcal{F}$-invariant and not contained in $\operatorname{sing}(\mathcal{F})$. Then

$$
\mathcal{P}_{n-k}(\mathbf{V}) \subset \mathcal{D}_{\mathcal{H}} \text { and } \mathcal{P}_{0}(\mathbf{V})=\mathbf{V} \not \subset \mathcal{D}_{\mathcal{H}}
$$

Proof. Let us first assume $\mathbf{V}$ is a linear subspace of $\mathbb{P}_{\mathbb{C}}^{n}$. In this case $\mathcal{P}_{j}=\emptyset$, for $j \geq 1$, so the first assertion of the lemma is meaningless. Assume then $\mathbf{V}$ is not a linear subspace and choose a pencil 
of hyperplanes $\mathcal{H}=\left\{H_{t}\right\}_{t \in \mathbb{P}_{\mathbb{C}}^{1}}$, with axis $L^{n-2}$ generic, so that $\operatorname{codim}\left(\mathcal{P}_{n-k}(\mathbf{V}), \mathbf{V}\right)=n-k$. If $q \in \mathcal{P}_{n-k}(\mathbf{V})$, then $\mathrm{T}_{q} \mathbf{V}$ meets $L^{n-2}$ in a subspace $W$ of dimension at least $n-k-1$. If $\mathrm{T}_{q} \mathbf{V} \subset L^{n-2}$ then any hyperplane $H_{t} \in \mathcal{H}$ contains $\mathrm{T}_{q} \mathbf{V}$, if not, a line $\ell \subset \mathrm{T}_{q} \mathbf{V}, \ell \not \subset L^{n-2}, \ell \cap W$ consisting of a point determines, together with $L^{n-2}$, a hyperplane $H_{t} \in \mathcal{H}$ such that $\mathrm{T}_{q} \mathbf{V} \subset H_{t}$. Since $\mathbf{V}$ is $\mathcal{F}$-invariant, we have $\mathrm{T}_{q} \mathcal{L} \subset \mathrm{T}_{q} \mathbf{V} \subset H_{t}$, in case $q$ is not a singular point of $\mathcal{F}$, where $\mathcal{L}$ is the leaf of $\mathcal{F}$ through $q$. This implies $q \in \operatorname{tang}\left(H_{t}, \mathcal{F}\right) \subset \mathcal{D}_{\mathcal{H}}$, so that $\mathcal{P}_{n-k}(\mathbf{V}) \subset \mathcal{D}_{\mathcal{H}}$. Also, it follows from the definition of $\mathcal{D}_{\mathcal{H}}$ that $\mathbf{V}$ is not contained in it.

Theorem I. Let $\mathcal{F}$ be a one-dimensional holomorphic foliation on $\mathbb{P}_{\mathbb{C}}^{n}$ of degree $d^{0}(\mathcal{F}) \geq 2$, with singular set of codimension at least 2 , and let $\mathbf{V}$ be an $\mathcal{F}$-invariant smooth irreducible algebraic variety, of dimension $n-k$, which is not a linear subspace of $\mathbb{P}_{\mathbb{C}}^{n}$, and not contained in $\operatorname{sing}(\mathcal{F})$. Suppose $\mathcal{P}_{n-k-j}(\mathbf{V}) \subset \mathcal{D}_{\mathcal{H}}$ but $\mathcal{P}_{n-k-j-1}(\mathbf{V}) \not \subset \mathcal{D}_{\mathcal{H}}$, for some $0 \leq j \leq n-k-1$. Then

$$
\frac{\varrho_{n-k-j}(\mathbf{V})}{\varrho_{n-k-j-1}(\mathbf{V})} \leq d^{0}(\mathcal{F})+1
$$

Proof. Observe that we may assume $\mathcal{P}_{n-k-j}(\mathbf{V}) \subset \mathcal{P}_{n-k-j-1}(\mathbf{V})$ and hence

$$
\mathcal{P}_{n-k-j}(\mathbf{V}) \subseteq \mathcal{D}_{\mathcal{H}} \cap \mathcal{P}_{n-k-j-1}(\mathbf{V})
$$

Bézout's Theorem then gives

$$
\varrho_{n-k-j}(\mathbf{V}) \leq\left(d^{0}(\mathcal{F})+1\right) \varrho_{n-k-j-1}(\mathbf{V})
$$

Corollary 1. Let $\mathbf{V}_{\left(d_{1}, \ldots, d_{k}\right)}^{n-k} \nsubseteq \operatorname{sing}(\mathcal{F})$ be a smooth irreducible complete intersection in $\mathbb{P}_{\mathbb{C}}^{n}$, which is not a linear subspace, defined by $F_{1}=0, \ldots, F_{k}=0$ where $F_{\ell} \in \mathbb{C}\left[z_{0}, \ldots, z_{n}\right]$ is homogeneous of degree $d_{\ell}, 1 \leq \ell \leq k$ and $\mathcal{F}$-invariant, where $\mathcal{F}$ is as in Theorem I. If $\mathcal{P}_{n-k-j}\left(\mathbf{V}_{\left(d_{1}, \ldots, d_{k}\right)}^{n-k}\right) \subset \mathcal{D}_{\mathcal{H}}$ but $\mathcal{P}_{n-k-j-1}\left(\mathbf{V}_{\left(d_{1}, \ldots, d_{k}\right)}^{n-k}\right) \not \subset \mathcal{D}_{\mathcal{H}}$ then

$$
d^{0}(\mathcal{F})+1 \geq \frac{\mathcal{W}_{n-k-j}^{(k)}\left(d_{1}-1, \ldots, d_{k}-1\right)}{\mathcal{W}_{n-k-j-1}^{(k)}\left(d_{1}-1, \ldots, d_{k}-1\right)}
$$

where $\mathcal{W}_{\delta}^{(k)}$ is the Wronski (or complete symmetric) function of degree $\delta$ in $k$ variables

$$
\mathcal{W}_{\delta}^{(k)}\left(X_{1}, \ldots, X_{k}\right)=\sum_{i_{1}+\cdots+i_{k}=\delta} X_{1}^{i_{1}} \ldots X_{k}^{i_{k}} .
$$

Proof. Immediate since $\varrho_{i}\left(\mathbf{V}_{\left(d_{1}, \ldots, d_{k}\right)}^{n-k}\right)=\left(d_{1}, \cdots . d_{k}\right) \mathcal{W}_{i}^{(k)}\left(d_{1}-1, \ldots, d_{k}-1\right)$.

Observe that if $\mathbf{V}$ is a smooth irreducible hypersurface, this reads $d^{0}(\mathcal{F})+2 \geq d^{0}(\mathbf{V})$. In (Soares 1997) we showed $d^{0}(\mathcal{F})+1 \geq d^{0}(\mathbf{V})$, but assumed $\mathcal{F}$ to be a non-degenerate foliation on $\mathbb{P}_{\mathbb{C}}^{n}$ 
Also, in (Soares 2000) the following estimate is obtained, provided $n-k$ is odd and $\mathbf{i}^{*} \mathcal{F}$ is non-degenerate: if $1 \leq k \leq n-2$ then

$$
d^{0}(\mathcal{F}) \geq \frac{\varrho_{n-k}\left(\mathbf{V}_{\left(d_{1}, \ldots, d_{k}\right)}^{n-k}\right)}{\varrho_{n-k-1}\left(\mathbf{V}_{\left(d_{1}, \ldots, d_{k}\right)}^{n-k}\right)}
$$

We remark that this estimate is sharper than that given in Corollary 1.

\section{THE TWO-DIMENSIONAL CASE}

As pointed out in Corollary 1, whenever we have a smooth irreducible $\mathcal{F}$-invariant plane curve $S$, the relation $d^{0}(S) \leq d^{0}(\mathcal{F})+2$ holds because $\varrho_{1}(S)=d^{0}(S)\left(d^{0}(S)-1\right)$, regardless of the nature of the singularities of $\mathcal{F}$, provided $\sin g(\mathcal{F})$ has codimension two.

In order to treat the case of arbitrary irreducible $\mathcal{F}$-invariant curves, let us recall the definition (see R. Piene 1978) of the class of a (possibly singular) irreducible curve $S$ in $\mathbb{P}_{\mathbb{C}}^{2}$. We let $S_{\text {reg }}$ denote the regular part of $S$ and, for a generic point $p$ in $\mathbb{P}_{\mathbb{C}}^{2}$, we consider the subset $\mathcal{Q}$ of $S_{\text {reg }}$ consisting of the points $q$ such that $p \in T_{q} S_{\text {reg. }}$. The closure $\mathcal{P}_{1}$ of $\mathcal{Q}$ in $S$ is the first polar locus of $S$, and the class $\varrho_{1}(S)$ of $S$ is its degree. $\mathcal{P}_{1}$ is a subvariety of codimension 1 whose degree is given by Teissier's formula (Teissier 1973):

$$
\varrho_{1}(S)=d^{0}(S)\left(d^{0}(S)-1\right)-\sum_{q}\left(\mu_{q}+m_{q}-1\right)
$$

where the summation is over all singular points $q$ of $S, \mu_{q}$ denotes the Milnor number of $S$ at $q$ and $m_{q}$ denotes the multiplicity of $S$ at $q$. Because $\mathcal{P}_{1}$ is a finite set of regular points in $S$, revisiting Lemma 3.1 we conclude:

$$
\mathcal{P}_{1} \subseteq \mathcal{D}_{\mathcal{H}} \cap S
$$

Also, $\operatorname{sing}(S) \subseteq \operatorname{sing}(\mathcal{F})$, so that

$$
\operatorname{sing}(S) \subseteq \mathcal{D}_{\mathcal{H}} \cap S
$$

and hence

$$
\mathcal{P}_{1} \cup \operatorname{sing}(S) \subseteq \mathcal{D}_{\mathcal{H}} \cap S
$$

It follows from Bézout's theorem that

$$
\varrho_{1}(S)+\sum_{q} m_{q} \leq\left(d^{0}(\mathcal{F})+1\right) d^{0}(S)
$$

Therefore we obtain the

THEOREM II. Let $S$ be an irreducible curve, of degree $d^{0}(S)>1$, invariant by a foliation $\mathcal{F}$ on $\mathbb{P}_{\mathbb{C}}^{2}$, of degree $d^{0}(\mathcal{F}) \geq 2$ with sing $(\mathcal{F})$ of codimension 2. Then

$$
d^{0}(S)\left(d^{0}(S)-1\right)-\sum_{q}\left(\mu_{q}-1\right) \leq\left(d^{0}(\mathcal{F})+1\right) d^{0}(S)
$$

where the summation extends over all singular points $q$ of $S$. 
This gives at once the following result, first obtained by Cerveau and Lins Neto (1991); COROLlaRY 2. If all the singularities of $S$ are ordinary double points (so that $\mu_{q}=1$ ) then

$$
d^{0}(S) \leq d^{0}(\mathcal{F})+2 .
$$

Theorem II illustrates one obstruction to solving Poincaré's problem in general, since we cannot estimate the sum $\sum_{q}\left(\mu_{q}-1\right)$ when dicritical singularities are present. However, if $S$ is an irreducible $\mathcal{F}$-invariant algebraic curve, which is a non-dicritical separatrix, then it follows from (Brunella 1997) that

$$
\sum_{q}\left(\mu_{q}-1\right) \leq \sum_{q} \sum_{i=1}^{r_{q}} G S V\left(\mathcal{F}, B_{i}^{q}, q\right)-\sum_{q} r_{q}
$$

where the sum is over all singular points $q$ of $S, B_{1}^{q}, \ldots, B_{r_{q}}^{q}$ are the analytic branches of $S$ at $q$, and $G S V$ denotes the Gomez-Mont/Seade/Verjovsky index.

REMARK. Let $S$ be a non-dicritical separatrix of $\mathcal{F}$, so that $d^{0}(S) \leq d^{0}(\mathcal{F})+2$. Assume equality holds in the expression in Theorem II, which amounts to

$$
d^{0}(S)\left(d^{0}(S)-d^{0}(\mathcal{F})-2\right)=\sum_{q}\left(\mu_{q}-1\right) \geq 0
$$

Hence we conclude $d^{0}(S)=d^{0}(\mathcal{F})+2$ and $S$ has only ordinary double points as singularities.

\section{$5 \mathcal{F}$-INVARIANT SMOOTH IRREDUCIBLE CURVES}

We have the following immediate consequence of Corollary 1: if we consider an $\mathcal{F}$-invariant smooth one-dimensional complete intersection $S=\mathbf{V}_{\left(d_{1}, \ldots, d_{(n-1)}\right)}^{n-(n-1)} \not \subset \operatorname{sing}(\mathcal{F})$, then

$$
d_{1}+\cdots+d_{n-1} \leq d^{0}(\mathcal{F})+n
$$

so that

$$
d^{0}(S) \leq\left(\frac{d^{0}(\mathcal{F})+n}{n-1}\right)^{n-1}
$$

provided codim $\operatorname{sing}(\mathcal{F}) \geq 2$. In the general case we have:

COROLlary 3. Let $S \nsubseteq \operatorname{sing}(\mathcal{F})$ be an $\mathcal{F}$-invariant smooth irreducible curve of degree $d^{0}(S)>1$, where $\mathcal{F}$ is a one-dimensional holomorphic foliation on $\mathbb{P}_{\mathbb{C}}^{n}$ of degree $d^{0}(\mathcal{F}) \geq 2$, with singular set of codimension at least 2. Then the first class $\varrho_{1}(S)$ of $S$ satisfies

$$
\varrho_{1}(S) \leq\left(d^{0}(\mathcal{F})+1\right) d^{0}(S)
$$

the geometric genus $g$ of $S$ satisfies

$$
g \leq \frac{\left(d^{0}(\mathcal{F})-1\right) d^{0}(S)}{2}+1 .
$$


Also, if $N(\mathcal{F}, S)$ is the number of singularities of $\mathcal{F}$ along $S$, then

$$
N(\mathcal{F}, S) \leq\left(d^{0}(\mathcal{F})+1\right) d^{0}(S) .
$$

Proof. Since $S$ is a curve which is not a line, we have to consider only $\varrho_{0}(S)=d^{0}(S)$ and $\varrho_{1}(S)$. The first inequality follows immediately from Theorem I. To bound the genus we observe that Lefschetz' theorem on hyperplane sections (Lamotke 1981) gives

$$
\varrho_{1}(S)=2 d^{0}(S)+2 g-2
$$

and the second inequality follows. On the other hand, since $S$ is irreducible and not contained in $\operatorname{sing}(\mathcal{F})$, Whitney's finiteness theorem for algebraic sets (Milnor 1968) implies that $S \backslash \operatorname{sing}(\mathcal{F})$ is connected, and hence $N(\mathcal{F}, S)$ is necessarily finite. Also,

$$
\operatorname{sing}(\mathcal{F}) \cap S \subset \mathcal{D}_{\mathcal{H}} \cap S
$$

and Bézout's theorem implies

$$
N(\mathcal{F}, S) \leq\left(d^{0}(\mathcal{F})+1\right) d^{0}(S)
$$

The first class of a smooth irreducible curve $S$ in $\mathbb{P}_{\mathbb{C}}^{n}$ was calculated by R. Piene (1976), and is as follows:

$$
\varrho_{1}(S)=2\left(d^{0}(S)+g-1\right)-\kappa_{0}
$$

where $g$ is the genus of $S$ and $\kappa_{0} \geq 0$ is an integer, called the $0-t h$ stationary index. It follows from Theorem I that:

COROLlary 4. With the same hypothesis of Corollary 3

$$
2 d^{0}(S)-\chi(S)-\kappa_{0} \leq\left(d^{0}(\mathcal{F})+1\right) d^{0}(S) .
$$

Remark on Extremal Curves. We can obtain an estimate for $d^{0}(S)$ in terms of $d^{0}(\mathcal{F})$ and $n \geq 3$, provided $S$ is non-degenerate (that is, is not contained in a hyperplane) and extremal (that is, the genus of $S$ attains Castelnuovo's bound). Recall that, for $S$ a smooth non-degenerate curve in $\mathbb{P}_{\mathbb{C}}^{n}$ of degree $d^{0}(S) \geq 2 n$, Castenuovo's bound is (Arbarello et al. 1985):

$$
g \leq \frac{m(m-1)}{2}(n-1)+m \epsilon,
$$

where

$$
d^{0}(S)-1=m(n-1)+\epsilon .
$$

The inequality

$$
g \leq \frac{\left(d^{0}(\mathcal{F})-1\right) d^{0}(S)}{2}+1
$$

together with $S$ extremal give, performing a straightforward manipulation:

$$
d^{0}(S) \leq 2\left(d^{0}(\mathcal{F})-1\right)(n-1)+\frac{(n-1)(n+2)}{n} .
$$




\section{ACKNOWLEGMENTS}

I'm grateful to M. Brunella for useful conversations, to PRONEX-Dynamical Systems (Brazil) for support and to Laboratoire de Topologie, Univ. de Bourgogne (France) for hospitality.

\section{RESUMO}

Consideramos o problema de relacionar carateres geométricos extrínsecos de uma variedade projetiva lisa e irredutível, que é invariante por uma folheação holomorfa de dimensão um de um espaço projetivo complexo, a objetos geométricos associados à folheação.

Palavras-chave: folheações holomorfas, variedades invariantes, classes polares, graus.

\section{REFERENCES}

Arbarello E, Cornalba M, Griffiths PA and Harris J. 1985. Geometry of Algebraic Curves, volume I. Grundlehren der mathematischen Wissenschaften 267, Springer-Verlag.

BRUnella M. 1997. Some remarks on indices of holomorphic vector fields. Publicacions Mathemàtiques 41: $527-544$.

CARniCer MM. 1994. The Poincaré problem in the non-dicritical case. An Math 140: 289-294.

Cerveau D and Lins Neto A. 1991. Holomorphic Foliations in $\mathbb{P}_{\mathbb{C}}^{2}$ having an invariant algebraic curve. An Institut Fourier 41(4): 883-904.

Fulton W. 1984. Intersection Theory, Ergebnisse der Mathematik und ihrer Grenzgebiete 3. Folge - Band 2, Springer-Verlag.

Lamotke K. 1981. The Topology of Complex Projective Varieties after S. Lefschetz. Topology 20: 15-51. Lins Neto A. 2000. Some examples for Poincaré and Painlevé problems. Pre-print IMPA.

MiLNOR J. 1968. Singular points of complex hypersurfaces. An Math Studies 61.

PIENE R. 1976. Numerical characters of a curve in projective n-space, Nordic Summer School/NAVF. Symposium in Mathematics, Oslo, August 5-25.

PIEne R. 1978. Polar Classes of Singular Varieties. An scient Éc Norm Sup 4e série 11: 247-276.

Poincaré H. 1891. Sur l'Intégration Algébrique des Équations Differentielles du Premier Ordre et du Premier Degré. Rendiconti del Circolo Matematico di Palermo 5: 161-191.

SoARes MG. 1997. The Poincaré problem for hypersurfaces invariant by one-dimensional foliations. Inventiones mathematicae 128: 495-500.

Soares MG. 2000. Projective varieties invariant by one-dimensional foliations. An Math 152: 369-382.

TeIssier B. 1973. Cycles évanescents, sections planes et conditions de Whitney. Astérisque 8-9: 285-362. 